\title{
In vivo imaging in mice reveals local cell dynamics and inflammation in obese adipose tissue
}

\author{
Satoshi Nishimura, ${ }^{1}$ Ichiro Manabe, ${ }^{1,2,3}$ Mika Nagasaki, ${ }^{1}$ Kinya Seo, ${ }^{4}$ \\ Hiroshi Yamashita, ${ }^{1}$ Yumiko Hosoya, ${ }^{1}$ Mitsuru Ohsugi, ${ }^{5}$ Kazuyuki Tobe, ${ }^{5}$ \\ Takashi Kadowaki, ${ }^{5}$ Ryozo Nagai, ${ }^{1}$ and Seiryo Sugiura ${ }^{4}$
}

\begin{abstract}
${ }^{1}$ Department of Cardiovascular Medicine and 2Nano-Bioengineering Education Program, University of Tokyo, Tokyo, Japan 3PRESTO, Japan Science and Technology Agency, Tokyo, Japan. ${ }^{3}$ Department of Human and Engineered Environmental Studies, Graduate School of Frontier Sciences, and ${ }^{5}$ Department of Metabolic Diseases, University of Tokyo, Tokyo, Japan.
\end{abstract}

\begin{abstract}
To assess physiological and pathophysiological events that involve dynamic interplay between multiple cell types, real-time, in vivo analysis is necessary. We developed a technique based on confocal laser microscopy that enabled us to analyze and compare the 3-dimensional structures, cellular dynamics, and vascular function within mouse lean and obese adipose tissue in vivo with high spatiotemporal resolution. We found increased leukocyte-EC-platelet interaction in the microcirculation of obese visceral adipose tissue in $o b / o b$ and highfat diet-induced obese mice. These changes were indicative of activation of the leukocyte adhesion cascade, a hallmark of inflammation. Local platelet activation in obese adipose tissue was indicated by increased P-selectin expression and formation of monocyte-platelet conjugates. We observed upregulated expression of adhesion molecules on macrophages and ECs in obese visceral adipose tissue, suggesting that interactions between these cells contribute to local activation of inflammatory processes. Furthermore, administration of anti-ICAM-1 antibody normalized the cell dynamics seen in obese visceral fat. This imaging technique to analyze the complex cellular interplay within obese adipose tissue allowed us to show that visceral adipose tissue obesity is an inflammatory disease. In addition, this technique may prove to be a valuable tool to evaluate potential therapeutic interventions.
\end{abstract}

\section{Introduction}

Despite significant advances in our understanding of the key role played by adipose tissue obesity in metabolic syndrome and cardiovascular disease, relatively little is known about the underlying cellular interplay that leads to adipose tissue dysfunction and systemic metabolic disturbance. Recent studies demonstrating infiltration of adipose tissue by macrophages and their secretion of inflammatory cytokines suggest the interplay between adipocytes and nonadipocytes is a contributor to adipose tissue pathology (1). Indeed, in vitro studies have shown that cross-talk between macrophages and adipocytes affects the function of both cell types (2-4), which highlights the need to analyze the cellular dynamics within obese adipose tissue in vivo. Unfortunately, the fragility of the large adipocytes that account for most of the volume of adipose tissue makes it difficult to preserve the integrity of the tissue under study. Consequently, our current models of the basic mechanisms governing adipose tissue function are mostly based on extrapolations from in vitro experiments or conventional histological analysis, and our understanding of these phenomena would greatly benefit from the availability of tools enabling in vivo observation and analysis. We therefore developed techniques for imaging real-time cellular dynamics with high spatiotemporal resolution in adipose tissue in living mice while maintaining tissue integrity by modifying the conventional in vivo confocal imaging method $(5,6)$. This system

Nonstandard abbreviations used: CLS, crown-like structure; HIF- $1 \alpha$, hypoxiainducible factor- $1 \alpha$; PI, propidium iodide; SVF, stromal-vascular fraction. Conflict of interest: The authors have declared that no conflict of interest exists. Citation for this article: J. Clin. Invest. 118:710-721 (2008). doi:10.1172/JCI33328. not only enabled acquisition of images of vasculature, leukocytes, erythrocytes, and platelets, in vivo, it also provided the means to evaluate indices of vascular function (i.e., blood flow and vascular permeability) and enabled observation of the effects of pharmacological intervention on cellular dynamics. We used this system to analyze inflammatory processes in obese adipose tissue.

Metabolic syndrome is increasingly recognized as a chronic, lowlevel, inflammatory state induced by obesity, and adipose tissue is considered to be a key site of interaction between adipocytes and other immune system effectors (1). We recently showed that adipose tissue obesity involves the coupling of adipogenesis and angiogenesis via close interactions among adipocytes, ECs, and stromal cells (7). In advanced obesity, the focal convergence of macrophages on necrotic adipocytes, designated crown-like structures (CLSs), is often found within adipose tissue. These structural alterations, along with increased expression of inflammatory cytokines, suggest that obese adipose tissue is a site of inflammation. However, it remains to be determined whether adipose tissue obesity also shows the fundamental features of inflammation, such as dynamic leukocyte-EC interactions.

Inflammation is a response to injurious stimuli in vascularized tissue and is initiated by recruitment of leukocytes from the bloodstream into the affected tissue (8). Leukocyte recruitment also plays essential roles in the perpetuation of inflammatory processes in chronic inflammatory diseases such as atherosclerosis. Although leukocyte recruitment can occur in larger atherogenic arteries, the microcirculation is the primary site of leukocyte recruitment in both acute and chronic inflammation (9). Leukocyte recruitment and migration are primarily controlled by 


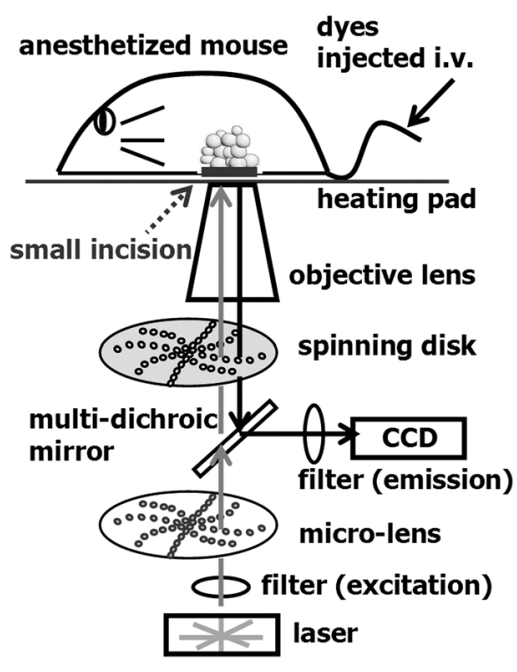

the interaction of leukocytes with vascular ECs and proceeds in several steps - i.e., tethering, rolling, activation, firm adhesion, and extravasation - mediated by adhesion molecules expressed on the surfaces of both cell types (10). Unstimulated leukocytes do not readily adhere to the vascular endothelium, but inflammatory signals induce the expression of proteins on EC and leukocyte surfaces that promote the adhesion and extravasation of activated immune cells from the circulation into the underlying tissues. The initial capture and rolling of leukocytes is mediated by selectin family proteins (P-selectin and E-selectin), and subsequent activation of integrins leads to their binding to ICAM-1 and VCAM-1 on the EC surface, resulting in firm adhesion (10). Finally, leukocytes infiltrate the extravascular space via PECAM-1. There is a good body of evidence showing increased levels of serum-soluble adhesion molecules, including soluble ICAM-1, VCAM-1, P-selectin, and E-selectin, in obese populations (11), and obesity is also associated with persistent platelet activation (12). These data suggest that obese adipose tissue is a site of enhanced interaction between ECs and leukocytes. However, to our knowledge, the dynamic cellular events taking place in the microcirculation in obese adipose tissue have not yet been directly analyzed.

Another important alteration in vascular function in obese adipose tissue is that blood flow per unit volume of tissue is diminished $(13,14)$. However, how the microcirculation is changed in obese adipose tissue and how multiple cell types interact with one another has not been directly investigated. To elucidate the dynamic changes in the microcirculation of obese adipose tissue and to address the functional and histological basis of adipose tissue inflammation, we examined the cellular dynamics of ECs, erythrocytes, leukocytes, and platelets in living adipose tissue in vivo using high-resolution, high-speed confocal microscopy. Our results demonstrate that the microcirculation and cellular dynamics were altered and that leukocyte recruitment was augmented in obese adipose tissue. Moreover, we found that obese visceral adipose tissue can be a local site of inflammation that may promote systemic inflammatory states in obese subjects.

\section{Results}

In vivo imaging system showing enhanced leukocyte-EC-platelet interaction and perturbed blood flow in obese visceral fat pads. To visually analyze the microcirculation and cellular dynamics within adipose tissue

\section{Figure 1}

Schematic diagram of the confocal microscope for real-time in vivo imaging. To visualize cell dynamics in vivo, fluorescent dyes were injected into the tail veins of anesthetized mice. A small incision was then made for an observation window, and the mouse was set on a heating pad to maintain body temperature. An inverted microscope equipped for Nipkow-spinning disk confocal laser microscopy, which enabled scanning at up to 1,000 frames/s, was used to visualize the tissue. See Methods for details.

in living mice, we developed in vivo imaging techniques (Figure 1) by modifying the methods that have previously been applied to other tissues $(5,6)$. The fat pads of anesthetized mice were observed through small dermal windows, without being exteriorized. Using a spinning-disk confocal microscope and a charge-coupled device camera, we visualized obese adipose tissue and assessed its cellular dynamics in living mice.

Blood cell dynamics were visualized by injecting FITC-dextran (Figure 2, A-I, and Supplemental Movies 1-6; supplemental material available online with this article; doi:10.1172/JCI33328DS1), and leukocytes were identified by nuclear staining with acridine orange (Figure 2, K, L, N, and O, and Supplemental Movies 7 and 8). Single-platelet kinetics were also visualized by injecting fluorescent anti-CD41 antibody (Supplemental Movie 9). We first compared epididymal adipose tissue in $o b / o b$ mice with that in lean control $o b /+$ mice. In $o b /+$ mice, capillary blood flow within epididymal adipose tissue was continuous, and leukocytes interacting with the vessel walls were rarely observed (Figure 2, D and G, and Supplemental Movies 1 and 4). But there was a striking difference in the obese epididymal adipose tissue of 12 -week-old $o b / o b$ mice: leukocytes rolling on $(o b /+, 1.85 \pm 0.58$ leukocytes $/ \mathrm{mm} ; o b / o b$, $10.30 \pm 2.67 ; n=5$, total 50 vessels; $P<0.01)$ or firmly adhering to the vessel walls $(o b /+, 1.03 \pm 0.45$ leukocytes $/ \mathrm{mm} ; o b / o b, 12.84 \pm 2.93$; $n=5$, total 50 vessels; $P<0.01)$ were frequently observed in postcapillary venules, which were identified based on vessel diameter and slower flow velocity than that seen in arterioles (Figure 2, K-P, and Supplemental Movies 7 and 8). Interestingly, firmly adhering leukocytes were often observed on vascular walls adjacent to CLSs (Figure 2O), which, as reported previously, consist of aggregated macrophages (3). In addition to leukocytes, adhesion of platelets to vessel walls also increased in postcapillary venules, and firm leukocyte adhesion was always coupled with platelet aggregation (Figure 2, E and N, and Supplemental Movies 2 and 8).

In contrast to epididymal fat pads, the inguinal subcutaneous fat pads of $o b / o b$ mice showed no significant increases in the levels of leukocyte rolling or adhesion in the microcirculation (Figure 2, M and $\mathrm{P}$ ). In addition, leukocyte-EC interactions were also not augmented in the skeletal muscle quadriceps femoris in $o b / o b$ mice. Thus in obesity, leukocyte-EC-platelet interactions are apparently selectively enhanced in visceral adipose tissue.

Further analysis of the blood flow within the epididymal adipose tissue revealed that while the blood flow in lean adipose tissue was largely continuous, even at the capillary level, the flow in obese epididymal adipose tissue was varied and often discontinuous (Figure 2, $\mathrm{G}$ and $\mathrm{H}$, and Supplemental Movies 4 and 5). The passing leukocytes appeared to perturb the flow in obese adipose tissue (Figure $2 \mathrm{H}$ and Supplemental Movie 5), and leukocytes firmly adhering to vessel walls were often observed at sites of discontinuous blood flow, suggesting that the leukocyte-EC interactions retard flow $(15,16)$. As a result, the average blood flow in the relatively small 

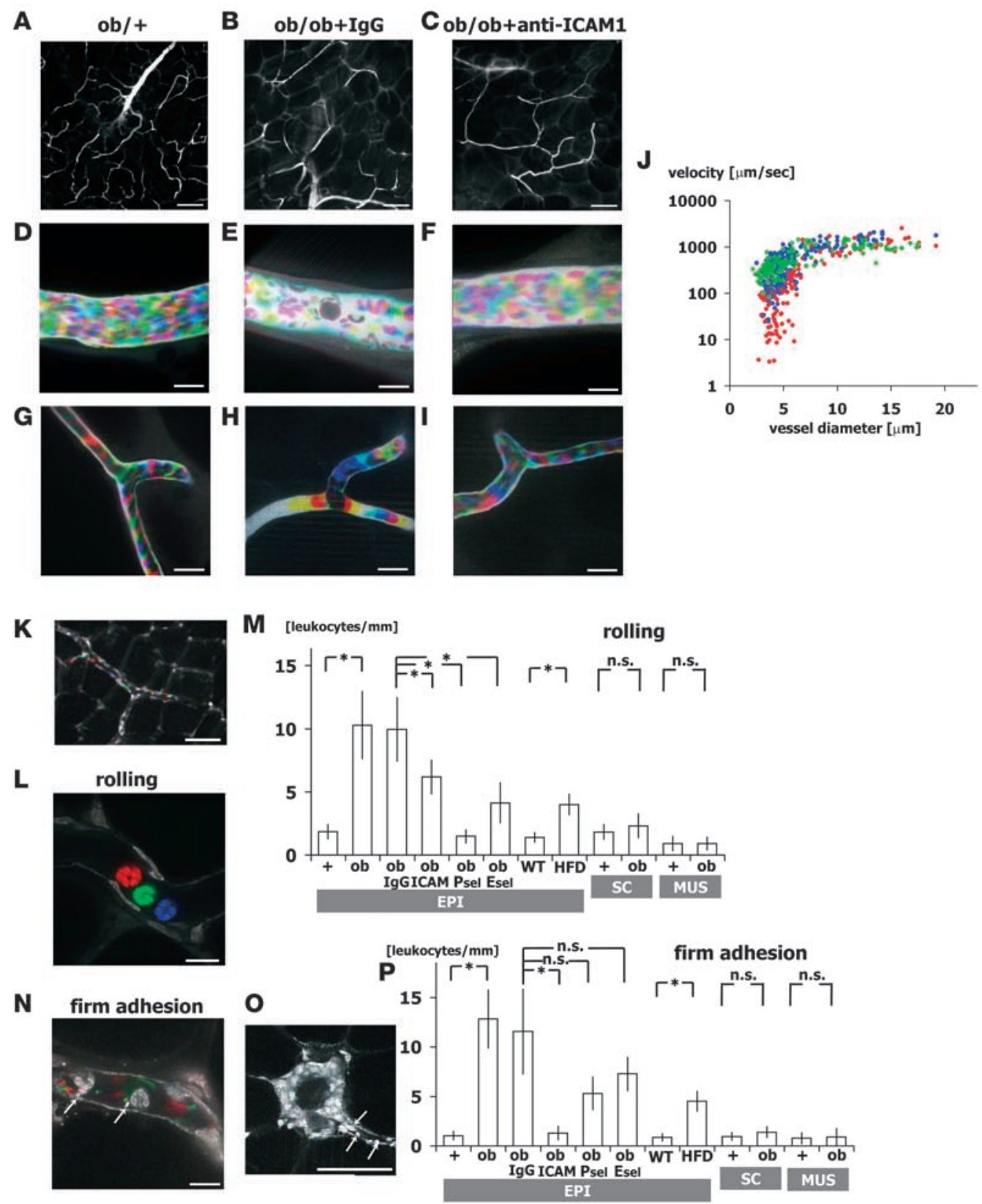

Q permeability

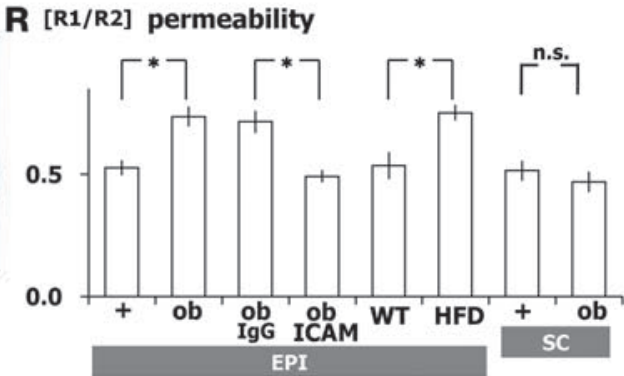




\section{Figure 2}

Abnormal leukocyte-EC-platelet interactions in obese adipose tissue revealed by intravital fluorescence microscopy. One-shot (A-C and $\mathbf{O})$ and time-lapse images (D-I, K, L, and $\mathbf{N}$ ) obtained by intravital fluorescence microscopy at the postcapillary venule (D-F) and capillary (G-I) levels in adipose tissue from ob/+, IgG-treated ob/ob, and anti-ICAM-1-treated ob/ob mice as indicated as well as untreated ob/ob mice $(\mathbf{K}, \mathbf{L}, \mathbf{N}, \mathbf{O}$, and $\mathbf{Q}$ ). Time-lapse images were reconstructed in red-green-blue order from 3 sequential images obtained at 30-ms intervals from Supplemental Movies 1-6 (D-I, respectively). Following FITC-dextran injection (MW 150,000), blood cells were negatively visualized in cyan-magenta-yellow order. Erythrocyte, leukocyte, and platelet cell dynamics were visualized at high spatiotemporal resolutions. Blood cell type was determined from the cell size. Where indicated, anti-ICAM-1, anti-E-selectin (Esel), anti-P-selectin (Psel), and normal rat IgG was injected intravenously prior to observation. Note the firmly adherent leukocytes and platelets on the vascular wall and the low hematocrit within the postcapillary venules of ob/ob mice (E). (J) Relationship between blood flow velocity and vascular diameter in adipose tissue from ob/+ (blue), lgG-treated ob/ob (red), and anti-ICAM-1-treated ob/ob (green) mice. Blood flow velocity at the capillary level $(<8 \mu \mathrm{m})$ was significantly slower in $o b / o b$ than $o b /+$ mice, but was increased to ob/+ levels by anti-ICAM-1 treatment $(n=5$, total 100 vessels/genotype). (K-P) Nuclear staining with acridine orange for specific visualization of leukocyte dynamics. Time-lapse images were reconstructed in red-green-blue order from 3 sequential images obtained at 90 -ms intervals from Supplemental Movies $7(\mathbf{L})$ and $8(\mathbf{N})$, and enabled leukocyte rolling and firm adhesion were visualized. FITC-conjugated anti-CD41 antibody was used to visualize platelet dynamics $(\mathbf{N})$. Number of rolling $(\mathbf{M})$ and firmly attached $(\mathbf{P})$ leukocytes per postcapillary venule length (leukocytes/mm; $n=5$, total 50 vessels/genotype) and in epididymal adipose tissue from lean C57BL/6 mice fed normal chow (WT) and high-fat diet-induced obese mice (HFD). In ob/ob mice, the number of rolling/adherent leukocytes significantly increased in epididymal fat (EPI), but not in inguinal subcutaneous fat (SC) or quadriceps skeletal muscle (MUS). ( $Q$ and $\mathbf{R}$ ) Vascular permeability of epididymal and subcutaneous adipose tissue analyzed using FITC-dextran (MW 4,000). Fluorescence intensities within the vessel (R2) and stromal space (R1) were measured, and R1/R2 ratio was used to assess the level of extravasation of FITC-dextran. Permeability increased in obese epididymal adipose tissue from ob/ob and high-fat diet-fed mice, but was normalized by anti-ICAM-1 ( $n=3$, total 60 points from 20 fields/genotype). Scale bars: $100 \mu \mathrm{m}(\mathbf{A}-\mathbf{C}$ and $\mathbf{K}), 50 \mu \mathrm{m}(\mathbf{O}), 10 \mu \mathrm{m}$ (D-I, L, N, and $\mathbf{Q}) .{ }^{*} P<0.05$.

capillaries $(<8 \mu \mathrm{m})$ was significantly slower in obese adipose tissue than in lean adipose tissue $(o b /+, 391 \pm 21 \mu \mathrm{m} / \mathrm{s}$; IgG-treated $o b / o b$, $187 \pm 17 \mu \mathrm{m} / \mathrm{s} ; n=5$, total 100 vessels; $P<0.01$; Figure $2 \mathrm{~J}$ ). Moreover, lower local hematocrits and slower blood flow velocities were seen in venules distal to capillaries with adherent leukocytes and platelet aggregations (Figure 2E). Such perturbation of blood flow was not observed in subcutaneous adipose tissue in $o b / o b$ mice, again indicating that leukocyte-EC interactions are selectively activated in visceral adipose tissue.

Our observation of local hypocirculation in obese epididymal adipose tissue is in agreement with previous reports (13) and implies local hypoxia. This was confirmed when we administered pimonidazole and observed increased formation of pimonidazole adducts in adipocytes and stromal cells in obese epididymal adipose tissue (Figure 3, A-D), which suggests that perturbation of the blood flow and the resultant hypoxia likely contribute to adipose tissue dysfunction in obesity. The hypoxic state of obese adipose tissue was supported by our finding there of upregulated expression of hypoxia-inducible factor-1 $\alpha$ (HIF-1 $\alpha$; Supplemental Figure 1).

Enhanced expression of adhesion molecules in ECs and macrophages in obese visceral adipose tissue. To investigate the molecular mechanisms underlying the heightened leukocyte-EC interactions within obese adipose tissue, we focused on expression of adhesion molecules by initially examining their expression levels in stromal-vascular fractions (SVFs) from epididymal fat pads (Figure 4, A-C). Real-time PCR analyses showed several-fold increases in the levels of ICAM-1, E-selectin, and P-selectin expression in SVFs from obese mice compared with control mice. To determine which cell populations within the SVFs contributed to the increases, surface expression of adhesion molecules and cell type-specific markers were analyzed by flow cytometry with gating to macrophages (F4/80 $\mathrm{D}-\mathrm{H})$ or ECs $\left(\mathrm{CD} 31^{+}\right.$; Figure $\left.4, \mathrm{I}-\mathrm{L}\right)$. When gated to ECs $\left(\mathrm{CD} 31^{+}\right.$ cells), surface expression of P-selectin, E-selectin, and ICAM-1 was increased in obese adipose tissue, whereas VCAM-1 expression was unchanged. That P- and E-selectins are not expressed under normal physiological conditions but are synthesized and exported to the EC surface under inflammatory conditions (17) indicates that the ECs are activated within obese adipose tissue.
We found that obesity also markedly affected expression of adhesion molecules on macrophages $\left(\mathrm{F} 4 / 80^{+}\right.$cells; Figure 4, $\mathrm{D}-\mathrm{F})$. As reported previously, the number of macrophages (defined as F4/80 ${ }^{+} \mathrm{CD} 11 \mathrm{~b}^{+}$cells) within SVFs was increased in obese adipose tissue $(o b /+, 11.7 \% \pm 1.7 \%$; ob $/ o b, 26.1 \% \pm 1.2 \%$; $n=5 ; P<0.01)$. When gated to macrophages (F4/80+ cells), the levels of ICAM-1 and L-selectin expression were significantly increased, whereas expression of CD18 (integrin $\beta 2$-chain, the ligand for ICAM-1) and CD162 (P-selectin ligand 1; PSGL1) was unchanged (Figure 4, G and H). Thus expression of adhesion molecules is clearly upregulated in both ECs and macrophages within obese adipose tissue.

Although ICAM-1 is expressed in leukocytes and ECs under normal conditions, it is upregulated by proinflammatory cytokines in atherosclerotic lesions (10). Robker et al. recently reported that expression of ICAM-1 protein is localized to ECs in lean adipose tissue (18), but to our knowledge its expression in obese adipose tissue has not previously been documented. Because our FACS analysis indicated that ICAM-1 expression was clearly upregulated in both ECs and macrophages (Figure 4, D and K), we further analyzed localization of ICAM-1 in adipose tissue using the confocal microscopy-based tissue imaging method that we recently developed (7). Immunohistochemical staining showed strong ICAM-1 and CCR2 expression in macrophages within CLSs in obese mice, suggesting M1 activated phenotypes (4), with stromal macrophages expressing lower levels of ICAM-1 (Figure 3, E-L).

Locally enhanced platelet dynamics in obese adipose tissue. That firmly adherent leukocytes were often found adjacent to CLSs (Figure 2O) suggests that the enhanced surface expression of adhesion molecules such as ICAM-1 likely promotes extravasation of macrophages into CLSs. We found that firm leukocyte adhesion was always coupled to platelet aggregation in obese epididymal fat pads (Figure 2, E and N, and Supplemental Movies 2 and 8). Indeed, platelet dynamics in postcapillary venules were markedly enhanced in obese adipose tissue, such that local aggregation and rolling of platelets on the vascular walls were significantly increased $(o b /+, 0.43 \pm 0.25$ adhered platelets $/ \mathrm{mm} ; o b / o b, 1.59 \pm 0.36$ adhered platelets $/ \mathrm{mm} ; n=5$, total 50 vessels; $P=0.01$; Supplemental 
research article
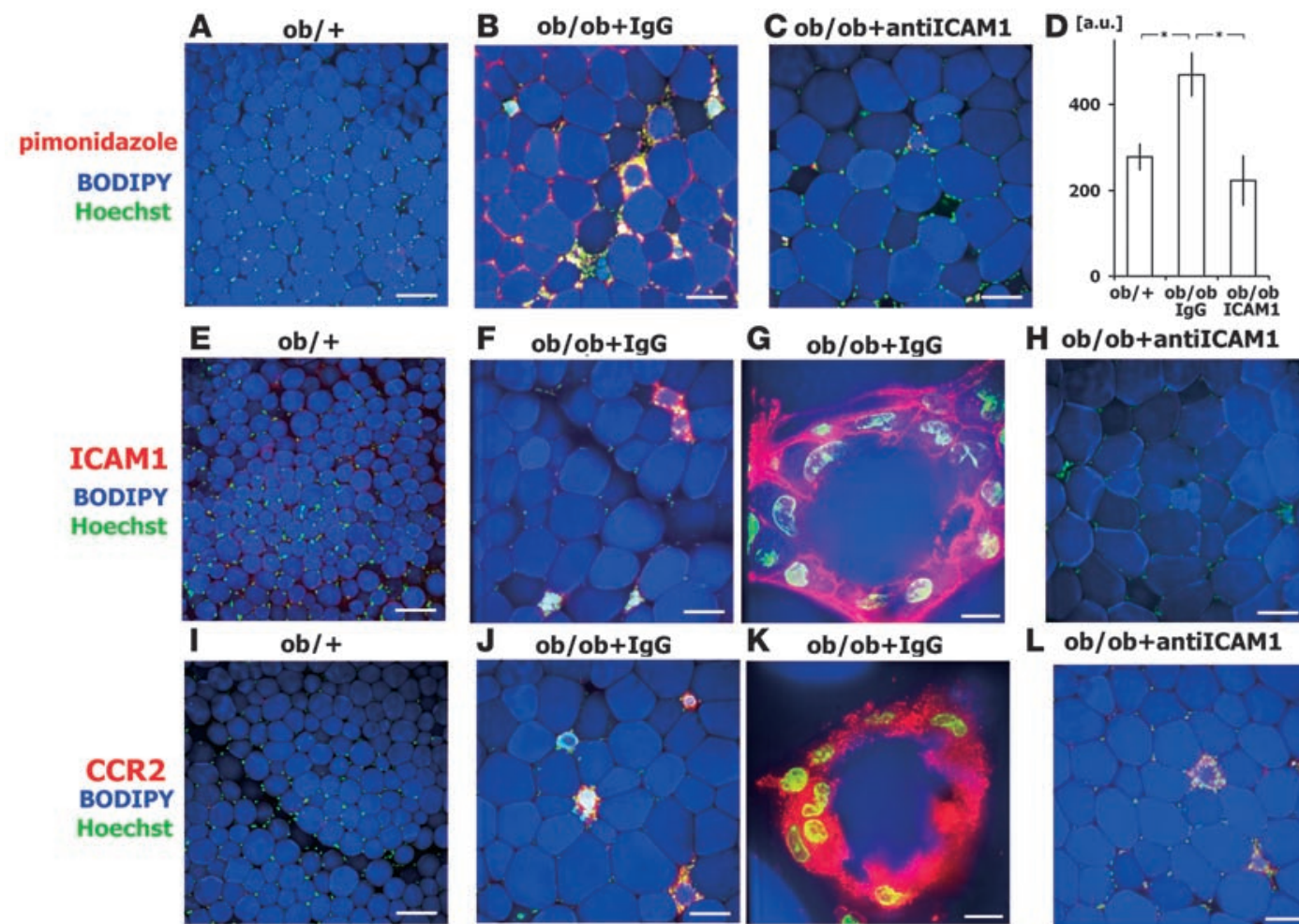

K ob/ob+IgG
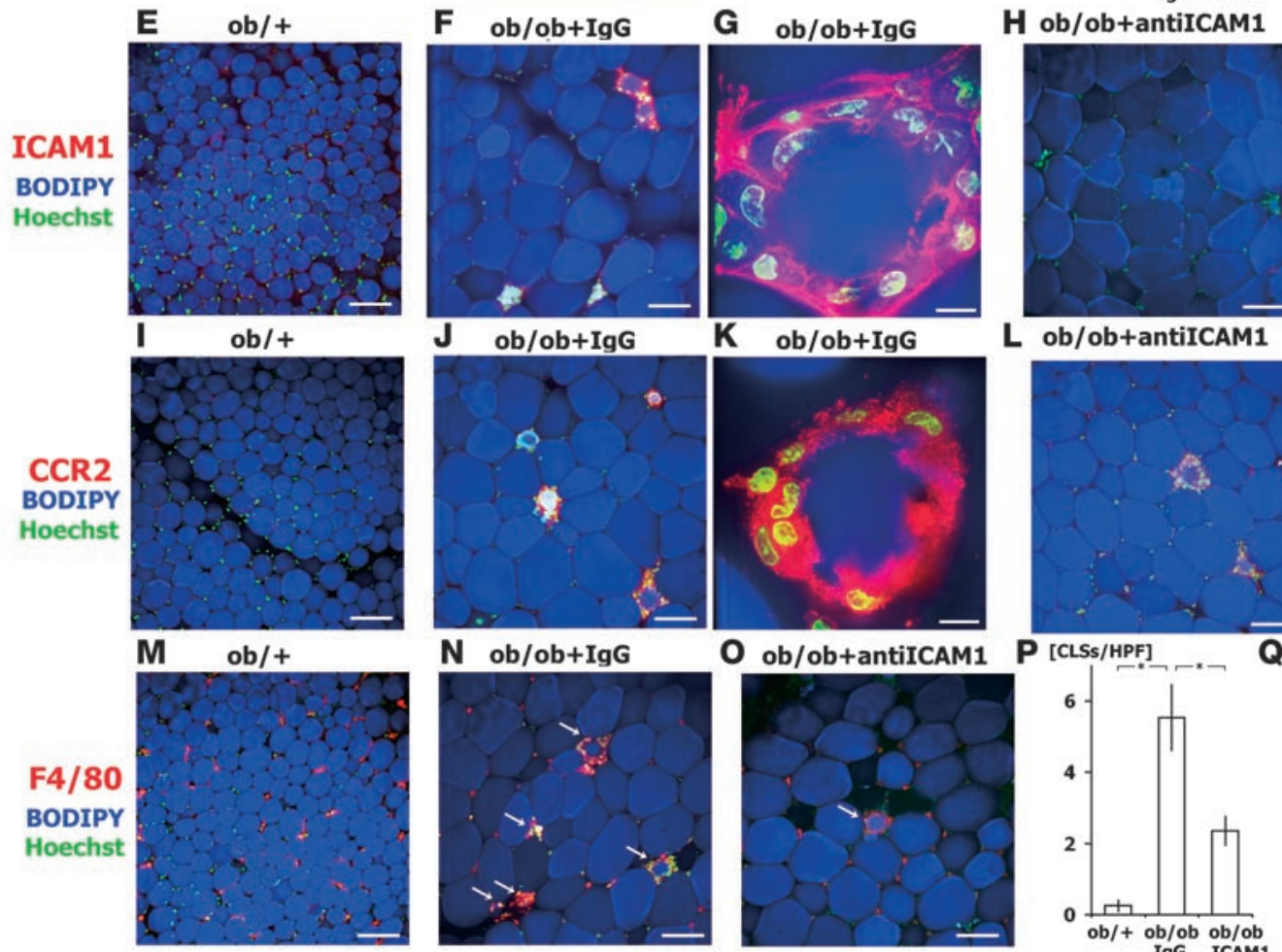

L ob/ob+antiICAM1
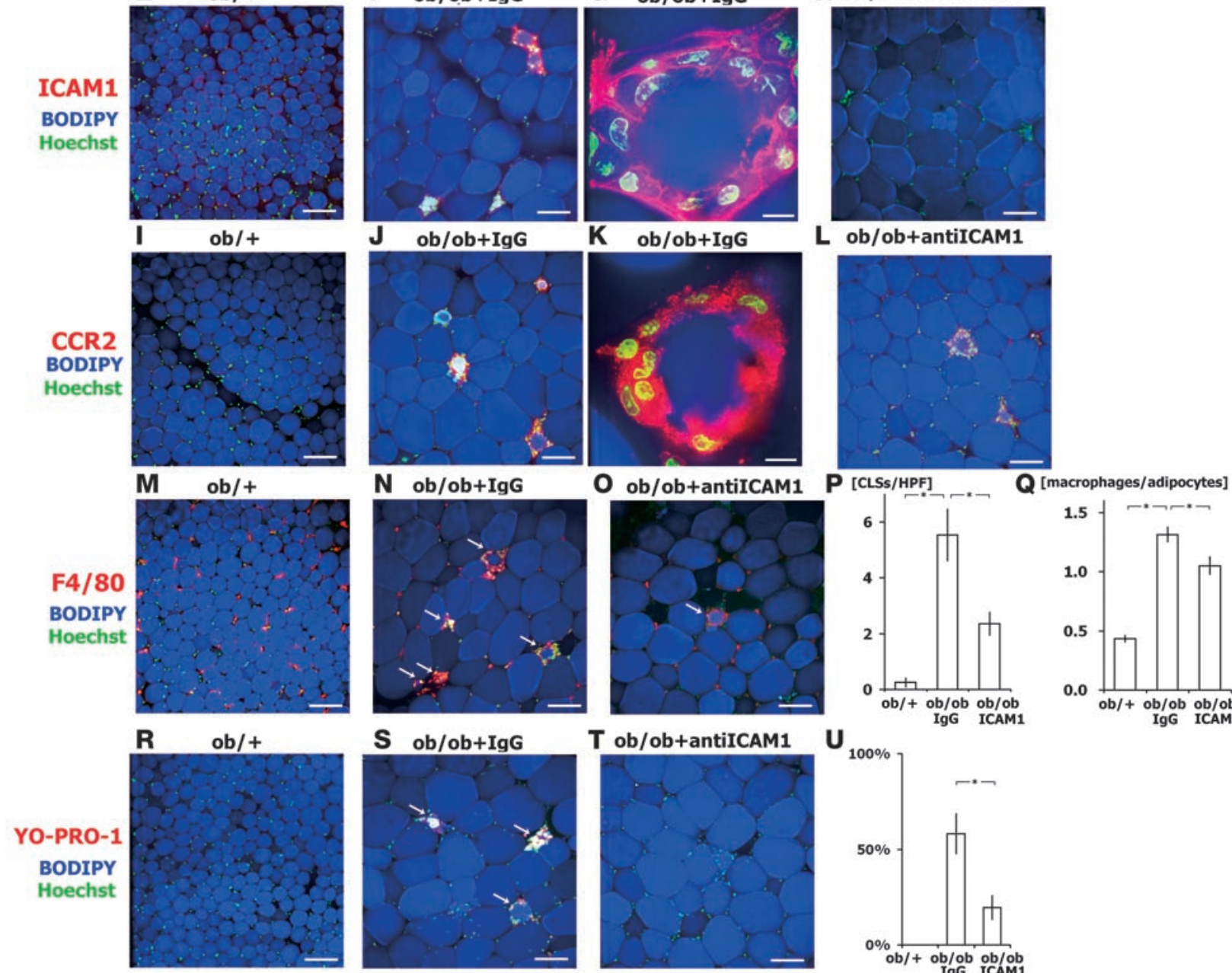

o ob/ob+antiICAM1
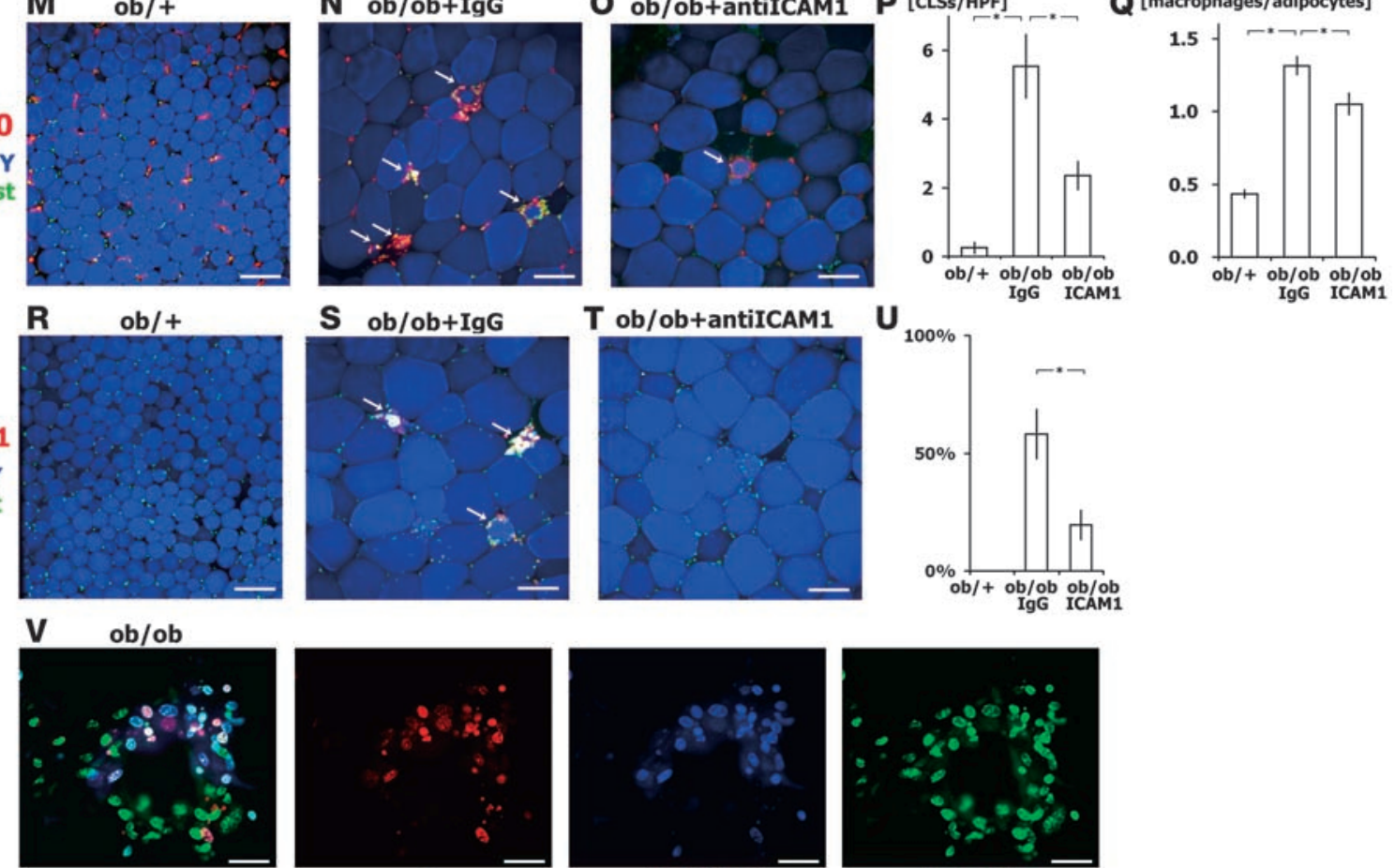

merge

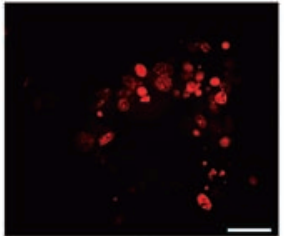

PI
T ob/ob+antiICAM1 $\mathbf{U}$
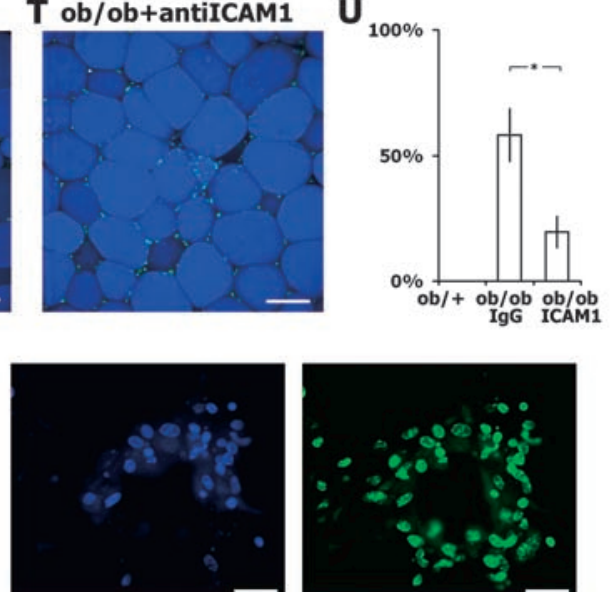

YO-PRO-1

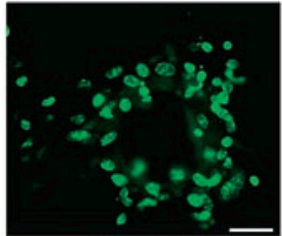

Hoechst 


\section{Figure 3}

CLS formation in adipose tissue in ob/ob mice. Epididymal adipose tissue obtained from 12-week-old ob/+, IgG-treated ob/ob, and antiICAM-1-treated ob/ob mice were examined with tissue imaging. Anti-ICAM-1 and control antibody was administered intraperitoneally (100 $\mu \mathrm{g} /$ mouse) 3 times per week for 2 weeks prior to observation. (A-D) Analysis of hypoxia using pimonidazole. Pimonidazole adducts were immunostained, adipocytes were counterstained with BODIPY, and nuclei stained with Hoechst. Adipose tissue from ob/ob mice was in a more hypoxic state than that from ob/+ mice, and anti-ICAM-1 mitigated the hypoxia. Pimonidazole adducts were found in both adipocytes and other cell types, particularly macrophages within CLSs. (D) The hypoxic state was quantified based on fluorescence intensity per low-power field $(n=5$, total 50 fields/genotype). (E-H) ICAM-1 staining showed that macrophages within CLSs strongly express ICAM-1 in IgG-treated ob/ob mice. (I-L) Macrophages within CLSs strongly express CCR2 in IgG-treated ob/ob mice, indicating macrophage activation within CLSs. (M-O) Immunohistochemical analysis of F4/80 staining showed increased CLS formation (arrows) in IgG-treated ob/ob mice. (P and $\mathbf{Q}$ ) Number of CLSs $(\mathbf{P})$ and macrophages $(\mathbf{Q})$ per number of adipocytes $(n=5$, total 1,000 adipocytes from 50 fields/genotype). Anti-ICAM-1 reduced CLS number. (R-T) Dead cell staining (YO-PRO1) of CLSs. Some CLSs contained dead adipocytes and macrophages that positively stained with YO-PRO1 (arrows). (U) CLSs with YO-PRO1-1+ cells relative to total CLS number ( $n=5$, total 50 fields/genotype). (V) Double staining with PI and $\mathrm{YO}-\mathrm{PRO} 1$ was performed to discriminate apoptotic $\left(\mathrm{YO}-\mathrm{PRO} 1^{+} \mathrm{PI}^{+}\right)$and necrotic $\left(\mathrm{YO}^{\mathrm{PRO}} 1^{+} \mathrm{PI}^{-}\right)$cell death in CLSs from ob/ob mice. Nuclei were counterstained with Hoechst. Scale bars: $100 \mu \mathrm{m}(\mathbf{A}-\mathbf{C}, \mathbf{E}, \mathbf{F}, \mathbf{H}-\mathbf{J}, \mathbf{L}-\mathbf{O}$, and $\mathbf{R}-\mathbf{T}), 10 \mu \mathrm{m}(\mathbf{G}, \mathbf{K}$, and $\mathbf{V})$. ${ }^{*} P<0.05$.

Movie 2). Furthermore, approximately $30 \%$ of the aggregated platelets were found with adherent leukocytes. To examine the activation status of systemic platelets, surface P-selectin expression was examined using flow cytometry with systemic blood obtained by cardiac puncture (Figure $5 \mathrm{~A}$ ). P-selectin is stored in $\alpha$-granules in resting platelets and expressed on the platelet surface only during and after platelet degranulation (19). We found no significant difference in surface P-selectin levels in platelets from the systemic circulations of lean and obese mice (Figure 5A). In contrast, platelets obtained from blood within adipose tissue showed markedly elevated surface expression of P-selectin, which is indicative of local platelet activation within obese adipose tissue (Figure 5B).

To further analyze platelet dynamics, we used flow cytometry to examine platelet-monocyte conjugate formation (Figure 5C). Degranulated platelets aggregate with monocytes, initially via the binding of platelet surface P-selectin to its PSGL1 counter-receptor on the surface of macrophages. Previous studies have shown that monocyte-platelet aggregates are highly sensitive indicators of platelet activation, particularly in vivo (20). Activated platelets have also been shown to interact with monocytes and the endothelium, depositing chemokines on their surfaces and promoting atherogenesis $(21,22)$. We counted monocyte-platelet aggregates in blood within adipose tissue using flow cytometry and found that the number of aggregates was markedly increased in obese adipose tissue. In addition, the number of circulating platelet-monocyte conjugates in the systemic circulation tended to be increased, but did not reach statistical significance, which is consistent with local activation of platelets within obese adipose tissue.

EC barrier dysfunction in obese visceral adipose tissue. Another hallmark of inflammatory responses in the microcirculation is EC barrier dysfunction (i.e., increased vascular permeability), which we evaluated based on the ratio of fluorescence intensities within the vessel and stromal space following injection of mice with small-sized FITCdextran (MW 4,000) $(23,24)$. We found that the FITC-dextran was highly extravasated into the stromal space in obese epididymal adipose tissue in $o b / o b$ mice, but not in control lean adipose tissue, indicating that vascular permeability was significantly increased in the former (Figure 2, Q and R). By contrast, we did not find increased vascular permeability in subcutaneous fat pads in $o b / o b$ mice.

Enhanced leukocyte-EC-platelet dynamics and EC barrier dysfunction in diet-induced obesity. To determine whether the observed enhancement of cellular dynamics is a fundamental feature of obese visceral adipose tissue, we analyzed the cellular dynamics in high-fat diet-induced obese mice. Body weights were significantly higher in 12-week-old mice fed a high-fat diet for 4 weeks than in mice fed normal chow (high-fat diet, $32.0 \pm 0.25 \mathrm{~g}$; normal chow, $25.6 \pm 0.25 \mathrm{~g}$; $n=5 ; P<0.01)$. The levels of leukocyte rolling and adhesion were significantly higher in epididymal adipose tissue in diet-induced obese mice than in control mice, although the increases were smaller than those observed in $o b / o b$ mice (Figure 2, M and P). We also observed increased vascular permeability in epididymal adipose tissue of mice with high-fat diet-induced obesity (Figure 2R). The results presented thus far clearly indicate that inflammatory changes are taking place in the microcirculation selectively within visceral obese adipose tissue. ECs, macrophages, and platelets are all locally activated and interacting via adhesion molecules.

Inhibition of adhesion molecules mitigates visceral adipose tissue inflammation. Levels of ICAM-1 expression were markedly increased on both macrophages and ECs in obese adipose tissue, and particularly high levels of ICAM-1 expression were present in macrophages within CLSs (Figure 3, E and F, and Figure 4, D and K). We also confirmed the presence of greater numbers of F4/80 cells (monocytes) expressing ICAM-1 in local adipose tissue blood (Figure 4M). Based on these findings, we hypothesized that inhibition of ICAM-1-mediated cellcell interactions might affect leukocyte-EC dynamics and inflammation in obese adipose tissue. To test that idea, we injected either anti-ICAM-1 neutralizing antibody or control normal IgG into $o b / o b$ mice. At $30 \mathrm{~min}$ after injection, the numbers of rolling (IgG-treated $o b / o b, 9.96 \pm 2.53$ leukocytes/mm; anti-ICAM-1 ob/ob, $6.18 \pm 1.33$ leukocytes $/ \mathrm{mm} ; n=5$, total 50 vessels; $P=0.03$ ) and adherent (IgG-treated $o b / o b, 11.56 \pm 4.28$ leukocytes $/ \mathrm{mm}$; anti-ICAM- $1 o b / o b, 1.30 \pm 0.71$ leukocytes $/ \mathrm{mm} ; n=5$, total 50 vessels; $P<0.01$ ) leukocytes were significantly diminished by anti-ICAM- 1 antibody compared with normal IgG (Figure 2, M and P). Administration of normal IgG did not significantly alter the levels of leukocyte rolling or adhesion compared with untreated $o b / o b$ mice (Figure 2, M and P). Anti-ICAM-1 treatment also mitigated the reduction in small capillary $(<8 \mu \mathrm{m})$ blood flow (IgG-treated $o b / o b, 187 \pm 17 \mu \mathrm{m} / \mathrm{s}$; anti-ICAM-1 $o b / o b$, $421 \pm 21 \mu \mathrm{m} / \mathrm{s} ; n=5$, total 100 vessels; $P<0.01$, Figure $2 \mathrm{~J})$. In addition, $24 \mathrm{~h}$ after administration of the anti-ICAM-1 antibody, vascular permeability had declined to a level similar to that seen in lean controls (Figure 2R). Thus, inhibition of ICAM-1 suppresses leukocyte-EC dynamics and improves EC function and blood flow.

Because surface expression of E- and P-selectins was increased in ECs, we also analyzed the effects of neutralizing antibodies against them. We found that the numbers of rolling leukocytes were significantly diminished by the antibodies. Similarly, the numbers of adherent leukocytes tended to be reduced by both $\mathrm{P}$ - and E-selectin blockade, but the difference did not reach statistical significance (Figure 2, M and P). 

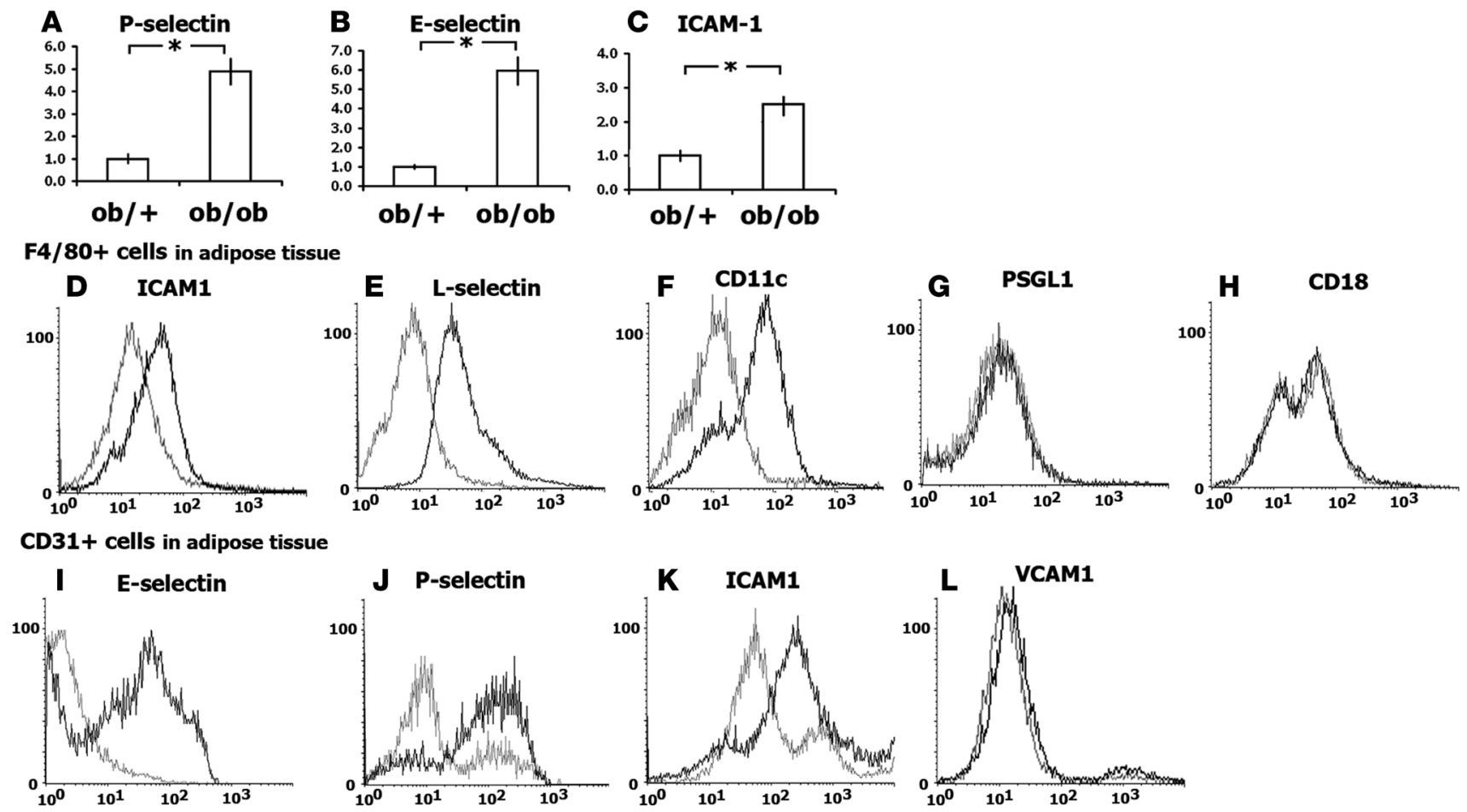

F4/80+ cells in local blood M ICAM1

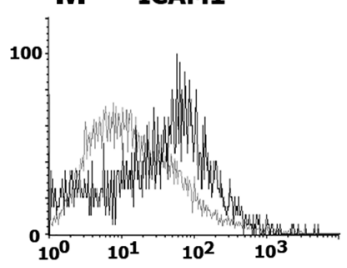

Figure 4

Enhanced surface expression of adhesion molecules in macrophages and ECs in obese adipose tissue. (A-C) Adhesion molecule expression examined by real-time PCR. mRNA expression of P- and E-selectin and ICAM-1 in the SVF of adipose tissue from ob/+ and ob/ob mice was analyzed $(n=5)$. (D-L) FACS analysis of the surface expression of adhesion molecules in F4/80+ macrophages (D-H) and CD31+ ECs (I-L) from $o b /+$ (gray line) and ob/ob (black line) mice. Expression of ICAM-1, L-selectin, and CD11c was upregulated in ob/ob compared with ob/+ macrophages. E-selectin, P-selectin, and ICAM-1 expression was upregulated in ob/ob ECs compared with ob/+ ECs. (M) Expression of ICAM-1 in $\mathrm{F} 4 / 80^{+}$cells was examined in local adipose blood. The $y$ axis denotes cell count; the $x$ axis denotes signal intensity. ${ }^{*} P<0.05$.

The observed acute effects of an ICAM-1 neutralizing antibody on leukocyte-EC dynamics prompted us to test whether chronic administration of the antibody further inhibits macrophage infiltration into adipose tissues. We administered the anti-ICAM-1 antibody or normal IgG 3 times per week for 2 weeks, after which the numbers of infiltrating macrophages and CLSs were determined. The number of CLSs was significantly higher in $o b / o b$ mice than $o b /+$ mice $(o b /+, 0.25 \pm 0.16 \mathrm{CLS} /$ field; IgG-treated $o b / o b$, $5.54 \pm 0.93 \mathrm{CLS} /$ field; $n=5$, total 50 fields; $P<0.01$; Figure 3, $\mathrm{M}-\mathrm{Q})$, as previously reported $(3,7,25,26)$. Treatment with antiICAM-1 antibody reduced CLS formation (anti-ICAM-1 ob/ob, $2.36 \pm 0.61 ; n=5$, total 50 fields; $P<0.01$ vs. IgG-treated $o b / o b$; Figure $3 \mathrm{P}$ ), and there was a corresponding reduction in the numbers of infiltrating macrophages $(o b /+, 0.43 \pm 0.03$ macrophages/ adipocyte; IgG-treated $o b / o b, 1.31 \pm 0.06$ macrophages/adipocyte; anti-ICAM-1 ob/ob, $1.05 \pm 0.07$ macrophages/adipocyte; $n=5$, total 1,000 adipocytes; $P<0.01$; Figure $3 \mathrm{Q}$ ).
We then determined whether CLSs contained dead adipocytes or macrophages. Previous studies have shown that adipocytes in CLSs undergo necrotic cell death (3). Only $26 \%$ of CLSs contained dead cells, including adipocytes and stromal cells (YO-PRO $1^{+}$cells), in anti-ICAM-1-treated mice, while 54\% of CLSs did so in control ob/ob mice (Figure 3, R-U). To distinguish apoptotic from necrotic cell death, double labeling with YO-PRO1 and propidium iodide (PI) was performed, and the results obtained showed almost $40 \%$ of the dead cells within CLSs $\left(\mathrm{YO}-\mathrm{PRO} 1^{+}\right.$) had undergone apoptotic cell death $\left(\mathrm{YO}-\mathrm{PRO}^{+} \mathrm{PI}^{+}\right.$) in obese mice (Figure $3 \mathrm{~V}$ ).

The reduction in macrophage infiltration was confirmed using flow cytometry based on counts of $\mathrm{F} 4 / 80^{+} \mathrm{CD} 11 \mathrm{~b}^{+}$cells (IgG-treated $o b / o b, 26.1 \% \pm 1.2 \%$ macrophages in SVF; anti-ICAM-1 ob/ob, $20.9 \% \pm 1.4 \%$ macrophages in SVF; $n=5 ; P<0.01$ ). Thus anti-ICAM-1 treatment not only inhibited leukocyte-EC interaction, it also mitigated macrophage infiltration into obese adipose tissue. 


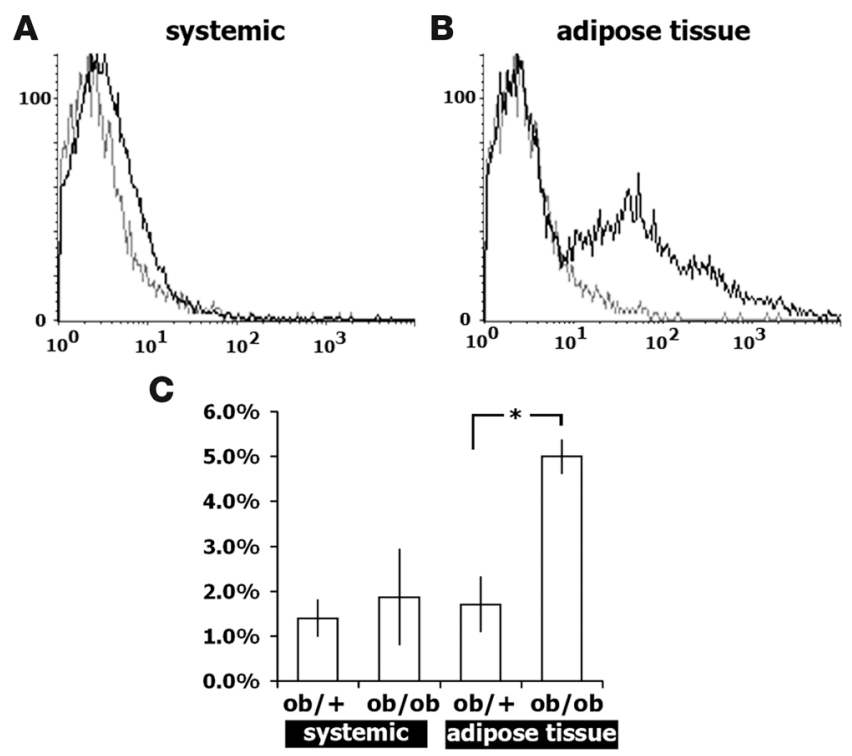

Figure 5

Increased platelet P-selectin expression and formation of monocyteplatelet conjugates in obese adipose tissue. (A and $\mathbf{B}$ ) Platelet surface $\mathrm{P}$-selectin expression in systemic circulation $(\mathbf{A})$ and local adipose blood (B) from ob/+ (gray line) and ob/ob (black line) mice was examined to evaluate the activation status of platelets. Platelets were gated based on CD41 expression and side scatter. Platelet activation was indicated in obese adipose tissue blood, but not in systemic blood from ob/ob mice. The $y$ axis denotes cell count; the $x$ axis denotes signal intensity. (C) Quantification of monocyte-platelet conjugates in the systemic circulation and adipose tissue blood. Systemic and local blood obtained from adipose tissue was examined by FACS using F4/80 (macrophage) and CD41 (platelet) gates. The ratio of the $\mathrm{F} 4 / 80^{+} \mathrm{CD} 41^{+}$ cell (conjugates) to the total $\mathrm{F} 4 / 80^{+}$cell count (macrophages) is shown ( $n=5$ per genotype). ${ }^{*} P<0.05$.

\section{Discussion}

Conventional techniques used to visualize tissue structure generally involve excision of the tissue, fixation, and sectioning; however, such techniques do not enable direct visualization of the complex cellular interplay taking place in living animals. Cellular reactions in inflammation are particularly dynamic and involve multiple cell types, and vascular circulation is absolutely essential for initiation of inflammatory responses. Consequently, analysis of the dynamic processes underlying inflammation requires in vivo imaging of intact tissues in living animals with high spatiotemporal resolution. Recent advances in optical instruments, including a spinning-disk confocal system and a high-speed scanning laser confocal microscope, have enabled high-speed confocal image acquisition. Confocal imaging has multiple advantages over conventional widefield microscopy. Higher spatial resolutions and thin optical slices can be obtained without being interfered by the out-of-focus haze of confocal microscopy. These are particularly important to visualize complex cellular dynamics. In earlier studies, intravital confocal microscopy was used to visualize the cellular dynamics with translucent tissues, including the mesentery and the cremaster muscle $(5,6)$. However, that technology is not directly applicable to adipose tissue, because it would require exteriorization of the fragile adipose tissue. In the present study we applied spinning-disk confocal microscopy, advanced image cap- ture methods, and fluorescent indicators to visualize intact living adipose tissue. Using a combination of fluorescent dextran and in vivo staining of blood cells with fluorescently labeled specific antibodies and fluorescent dyes, we visualized - for the first time to our knowledge - the cellular dynamics within intact living adipose tissue. The results presented clearly establish obese adipose tissue as a site of active inflammation; moreover, the observed mitigation of inflammation by specific anti-adhesion molecule antibodies illustrates the utility of this technology for evaluating new approaches to treating adipose tissue abnormalities.

Previous studies have shown that obese adipose tissue secretes various inflammatory mediators (e.g., TNF- $\alpha$ and IL-6), as well as adipokines such as adiponectin, and suggest that adipose tissue plays a crucial and integral role in systemic inflammation in obese subjects. Subsequent findings of augmented infiltration of macrophages into obese adipose tissue (25) and the vicious interactions between adipocytes and macrophages via inflammatory cytokines and lipids (2) suggested the inflammatory changes seen in obese adipose tissue may be the key pathology that promotes systemic inflammatory states in obese subjects. Still, little was known about how inflammation is initiated and what inflammatory changes take place within obese adipose tissue. Our present findings clearly demonstrate that adipose tissue obesity involves interactions among activated ECs, leukocytes, and platelets. Obese adipose tissue is also subject to reduced blood flow velocity and increased hypoxia. These dynamic changes are common and fundamental features of inflammation. Thus, by visualizing the cellular dynamics, we clearly established obese adipose tissue as a site of inflammation and determined that local events taking place within the adipose tissue microcirculation play an essential role in the initiation and progression of adipose tissue inflammation.

Our findings also demonstrate that the leukocyte adhesion cascade is activated in obesity and plays an essential role in in situ inflammation within obese visceral adipose tissue. The accumulation of lipid-loaded cells under the endothelium is a hallmark of early-stage atherosclerotic lesions and is initiated by the interaction of activated ECs and leukocytes (27), which means that similar mechanisms are operating during the initiation of inflammation in adipose tissue and arteries. Recently, we demonstrated that angiogenesis is indispensable to the progression of adipose tissue obesity (7). Likewise, angiogenesis within the arterial wall is important for the progression of atherosclerosis (28). Cellular interactions between resident cells and macrophages via inflammatory cytokines and other factors are actively taking place in both atherosclerotic plaques and obese adipose tissue (2), which suggests that a number of common mechanisms underlie both atherosclerosis and adipose tissue obesity. It is now established that atherosclerosis is a chronic inflammatory disease (29). Chronic inflammation is characterized by infiltration of mononuclear cells, tissue destruction, and healing through replacement of the connective tissue in the damaged area, which is accomplished through angiogenesis and, especially, fibrosis (30). But closely linked concomitant progression of tissue destruction and healing will lead to rearrangement of the original architecture or remodeling of the tissue (31). Although we obtained no clear evidence of fibrosis in adipose tissue in the obese mouse models we used, fibrosis does reportedly occur in adipose tissue in KKAy mice under certain conditions $(\mathrm{H}$. Sakaue, Kinki University, Higashiosaka, Japan, personal communication). Moreover, we observed that in $o b / o b, d b / d b$, and high-fat diet-induced obese mice, adipose tissue obesity involves dynamic 
structural changes, including adipocyte hypertrophy, angiogenesis, CLS formation, adipogenesis, stromal cell proliferation, and adipocyte death $(3,7,25)$. In addition, previous studies have shown that certain MMPs are activated in obese adipose tissue $(32,33)$ and that inhibition of MMPs mitigate adipose tissue obesity $(34,35)$. Consistent with those findings, adipocytes are enveloped by a basement membrane, and only after its extensive remodeling can adipocyte hypertrophy occur (36). Moreover, adipogenesis is closely coupled with angiogenesis and requires cooperative growth of stromal cells, which also requires remodeling of the extracellular matrix (7). These dynamic changes in the organization of adipose tissue architecture can be considered adipose tissue remodeling and very likely use the same molecular mechanisms that promote tissue remodeling in chronic inflammation of other tissues. When compared with other chronic inflammatory diseases, such as atherosclerosis, liver fibrosis, and asthma, the features of visceral adipose tissue obesity, including tissue remodeling, activated leukocyte-platelet-EC interactions, and active involvement of macrophages, collectively indicate that visceral adipose tissue obesity can be considered to be chronic inflammatory disease.

As previously reported $(37,38)$, much less macrophage infiltration was observed in subcutaneous fat in obese mice, although the adipocytes exhibited a degree of hypertrophy that was similar to that seen in epididymal fat (data not shown). In contrast to epididymal fat, obese subcutaneous fat did not exhibit enhanced leukocyte-EC interactions or vascular permeability (Figure 2), which is consistent with previous findings that visceral fat expresses higher levels of inflammatory cytokines and contains a greater number of macrophages than does subcutaneous fat $(37,38)$. In addition, numerous clinical studies have established that visceral obesity is a more important risk factor for cardiovascular disease than subcutaneous obesity (39). Differential activation of the leukocyte adhesion cascade also suggests that, in addition to the systemic changes involved in obesity, local factors within the epididymal fat play an important role in the inflammatory response, an idea supported by our observation of local activation of platelets (Figure 5). These local factors may be the reason there is greater association between visceral obesity and cardiovascular risk.

Analysis of the effects of anti-adhesion molecule antibodies demonstrated the analytical power of our imaging technology and that leukocyte-EC interactions are potential therapeutic targets in the treatment of obesity and adipose tissue dysfunction. Acute inhibition of ICAM-1 using an anti-ICAM-1 antibody suppressed leukocyte adhesion and vessel hyperpermeability and improved blood flow, demonstrating that the leukocyte-EC interaction is crucial for activation of local inflammatory processes and that ICAM-1 is an important inflammatory mediator in adipose tissue. The importance of the leukocyte-EC interaction in inflammation was further substantiated by our experiments using anti-E-selectin and anti-P-selectin neutralizing antibodies. Interestingly, chronic administration of anti-ICAM-1 antibody suppressed formation of CLSs, which are typically found in advanced cases of adipose tissue obesity $(3,7)$. The macrophages contained in CLSs exhibit the classical CCR2 ${ }^{+} \mathrm{M} 1$ activated macrophage phenotype (Figure 3, J and $\mathrm{K})$ and are known to produce various inflammatory cytokines and reactive oxygen species (4) and to engulf dead adipocytes within the CLSs (3). We found that macrophages within CLSs were also positive for dead cell markers (Figure 3, R-V). Almost $40 \%$ of the dead macrophages within CLSs in $o b / o b$ epididymal fat had undergone apoptotic cell death (Figure $3 \mathrm{~V}$ ). The precise underly- ing mechanism is unclear, but it is tempting to speculate that after macrophages engulf necrotic adipocytes, they undergo apoptotic cell death to avoid unnecessary and consistent inflammation (40, 41). The reduction of macrophages in fat pads by anti-ICAM-1 treatment also implies that there is some remodeling of resident macrophage numbers. However, we could not determine the residence lifetime because of the lack of markers that distinguish resident macrophages from macrophages that infiltrate from the systemic circulation. These results of the chronic anti-ICAM-1 treatment indicate that leukocyte-EC interaction is necessary not only for initiation of inflammation, but also for progression of chronic inflammatory changes.

We also found that blood flow velocity was slowed in obese adipose tissue, causing it to be in a hypoxic state that likely contributes to the metabolic dysfunction within the tissue (42). To rule out the possibility that altered rheological properties of erythrocytes in obese mice might have affected the microcirculation, we performed an indentation test by combining atomic force microscopy and confocal microscopy. The results showed no significant differences in the mechanical properties of erythrocytes in lean and obese mice (data not shown). That the slowed blood flow was most apparent in capillaries containing adherent leukocytes and platelet aggregates suggests that the slowing was a result of enhanced leukocyte-EC interaction, but it remains difficult to draw conclusions as to whether the leukocyte-EC interaction or the retardation of blood flow that initiated the changes we observed in the adipose microcirculation. This is because low venular shear force caused by reduced blood flow reportedly promotes leukocyte-EC-platelet interactions (43), and the resultant leukocyte adhesion increases network resistance (44), perturbing blood flow. This vicious cycle likely augments leukocyte-EC interactions and inflammatory states.

Another important finding was that vascular permeability increased in obese visceral fat and was normalized by anti-ICAM-1 treatment. Capillary permeability can be altered by several mechanisms, including nitric oxide synthesis, a change in the prostanoid balance, and increased oxidative stress $(23,24)$. Leukocyte adhesion to ECs also can trigger vascular leakage $(45,46)$. The finding that anti-ICAM-1 treatment reduced vascular permeability (Figure 2R) suggests that leukocyte-EC interactions may play an important role in increasing vascular permeability. On the other hand, during the initiation of atherosclerosis, it is endothelial dysfunction - including hyperpermeability and retention of modified lipoproteins - that is the crucial step triggering the leukocyte adhesion cascade and inflammation (47). Further study will be needed to identify what initiates the cascade of inflammatory responses and the order of events in adipose tissue obesity. In order to address these important questions, it will be necessary to combine in vivo visualization technology with genetic and pharmacological interventions. Our present results provide a strong basis for these studies as well as for the development of novel therapeutic strategies.

\section{Methods}

Mice. Twelve-week-old $o b / o b$ and $o b /+$ mice were obtained from Charles River Inc. To examine the chronic effects of ICAM-1 blockade on adipose tissue structure, purified rat neutralizing anti-mouse ICAM-1 antibody was intraperitoneally administered $(100 \mu \mathrm{g} /$ mouse, clone YN1/1.7.4; Biolegend) 3 times per week for 2 weeks prior to experimentation; the control $o b / o b$ group received nonspecific IgG (100 $\mu \mathrm{g} / \mathrm{mouse}$; SigmaAldrich) in a similar manner. 
Diet-induced obese mice were prepared as follows: 8-week-old C57BL/6 mice were purchased from Charles River Inc., housed under a 12-h light/12-h dark cycle, and allowed free access to standard laboratory food (6\% fat; Oriental Yeast) or high-fat food (32\% fat; CLEA Japan). After 4 weeks on the diet, the cell dynamics were examined in the 12 -week-old mice.

To examine the acute effects of ICAM-1, E-selectin, and P-selectin blockade on cell dynamics and vascular permeability, neutralizing rat anti-mouse E-selectin $(2 \mu \mathrm{g} / \mathrm{g}$ body wt, clone 10E9.6; Research Diagnostics), P-selectin ( $2 \mu \mathrm{g} / \mathrm{g}$ body wt, clone R40.34; Research Diagnostics) or ICAM-1 (2 $\mu \mathrm{g} / \mathrm{g}$ body wt, clone YN1/1.7.4; Biolegend) antibody was injected via the tail vein before the in vivo imaging experiments. In addition, to rule out the effect of nonspecific IgG injection ( $2 \mu \mathrm{g} / \mathrm{g}$ body wt) into $o b / o b$ mice, we repeated the leukocyte cell dynamics analysis in $o b / o b$ mice without antibody injection. All of the antibody clones used were validated in the in vivo and in vitro neutralizing studies (48-50). All experiments were approved by the University of Tokyo Ethics Committee for Animal Experiments and strictly adhered to the guidelines for animal experiments of the University of Tokyo.

Preparation of mice for in vivo imaging. To visualize cellular dynamics in vivo, mice were anesthetized by injection with urethane $(1.5 \mathrm{~g} / \mathrm{kg})$. Our preliminary studies showed that urethane was most suitable to use because in contrast to pentobarbital, isoflurane, barbiturate, and ketamine-xylazine, it did not significantly affect the local microcirculation in the adipose tissue. After the appropriate depth of anesthesia was obtained, a small incision was made and moistened with saline. This window was then covered with Saran Wrap. Intravital imaging was performed through the small ( $\sim \mathrm{mm})$ window without exteriorizing the fat pad as shown in Figure 1. Heating pads were used to keep the body temperature at $37^{\circ} \mathrm{C}$.

Dye and antibody injections to visualize cell dynamics. To visualize blood flow and blood cells, mice were infused with FITC-dextran $(5 \mathrm{mg} / \mathrm{kg}$ body wt, MW 150,000) via the tail vein, and blood cells were visualized as dye-free elements. We were able to discriminate erythrocytes, leukocytes, and platelets based on their size and deformation in high-power field images. To visualize leukocyte nuclei, acridine orange dye was administered $10 \mathrm{~min}$ before the experiments $(1.25 \mathrm{mg} / \mathrm{kg}$ body wt). To specifically visualize single-platelet kinetics, R-phycoerythrin-conjugated anti-CD41 antibody (BD Biosciences - Pharmingen) was injected 20 min before experiments $(0.1 \mu \mathrm{g} / \mathrm{g}$ body wt). The concentration used in our experiments was lower than the value previously shown to have no significant impact on in vivo leukocyte-EC interactions (51).

Microscope setup for in vivo imaging. The experimental set up for in vivo imaging is shown in Figure 1. We used an inverted microscope (IX71; Olympus) equipped for Nipkow spinning-disk confocal microscopy (CSU22; Yokogawa-denki) to obtain images with high time and spatial resolution $(80 \mathrm{~nm} / \mathrm{pixel})$. The system consisted of a rotating microlens array that efficiently guided laser beams into pinholes and a spinning disk. The disks were rotated as rapidly as 5,000 rpm, which enabled the acquisition of images at up to 1,000 frames/s.

An electron multiplying charge-coupled device (EMCCD; Impactron CCD; Nihon TI) was used to obtain sequential images because it offers excellent sensitivity and high signal-to-noise ratio. All sequential images were obtained at $30 \mathrm{frames} / \mathrm{s}$ with the exposure time of $10 \mathrm{~ms}$ for each image. The short exposure time was essential for visualization of the cellular dynamics in the microcirculation. In the preliminary experiment using a high-speed C-MOS camera and image intensifier (SV-200i; Photron), we were able to acquire images at 1,000 frames/s. However, acquired images were far inferior to the ones obtained using the EMCCD in terms of spatial resolution. Because the high spatial resolution is vital for analyzing cellular interactions and the speed of 30 frames/s was sufficient to visualize cell dynamics in capillaries and postcapillary venules, we used the EMCCD camera throughout the present study. The images were obtained sequen- tially, without averaging, and quantitative analysis was performed by blinded observers using IpLab software version 3.6 (Scanalytics).

Our system is equipped with an argon-krypton laser for excitation at $\lambda$ excitation 488 or $568 \mathrm{~nm}$, and the emission signal was collected using a multidichroic mirror and appropriate band-pass filters. However, due to the long changing time of mechanical filters (Ludl Electronic Products Ltd.), we could obtain monochromatic videos with single excitation.

A high-power object lens ( $\times 100$ oil, numerical aperture 1.40, UPlanSApo; Olympus) was used mainly to visualize cell dynamics, including those of erythrocytes and platelets. A low-power lens ( $\times 10$ dry, numerical aperture 0.30 , UPlanFL; Olympus) was used to visualize adipose tissue structure. Because a high numerical aperture was critical for in vivo visualization, lenses with long working distances were inappropriate for our purpose.

Quantification of leukocyte dynamics. To quantify leukocyte dynamics, sequential images were obtained and analyzed as follows. Rolling leukocytes were defined as those that showed continuous interaction with the endothelium when moving through the vessels. Firmly adhering leukocytes were defined as those adhering to the endothelium and remaining within a $20-\mu \mathrm{m}$ segment of vessel for more than $30 \mathrm{~s}$. The numbers of rolling and adherent leukocytes were counted in vessels in randomly selected fields, averaged, and expressed as the number of leukocytes per mm (vessel length).

Quantification of vascular permeability. To quantify vascular permeability, mice were injected with small-sized FITC-dextran (MW 4,000) via the tail vein, and images of the vessels were obtained beginning $10 \mathrm{~min}$ after injection. The fluorescence intensities within the vessel (R2) and the stromal space (R1) were then measured, after which the R1/R2 ratio was used to assess the level of extravasation (Figure 2Q).

Tissue imaging ex vivo. For immunohistochemical analyses, mice were sacrificed by cervical dislocation, after which the epididymal fat was removed using a sterile technique and minced into small pieces $(\sim 2-3 \mathrm{~mm})$ using a scalpel. The tissue pieces were washed, fixed in $4 \%$ formaldehyde for $45 \mathrm{~min}$, permeabilized with $1 \%$ Triton X-100 (Calbiochem) for $10 \mathrm{~min}$, and blocked with $1 \%$ bovine serum albumin. The specimens were then incubated first for $12 \mathrm{~h}$ with a primary antibody (anti-F4/80, Caltag; anti-ICAM-1, Santa Cruz Biotechnology; anti-CCR2, Abcam) at 1:100-1:200 dilution and then for $1 \mathrm{~h}$ with Alexa Fluor 488-conjugated secondary antibody (Invitrogen) at $1: 100$ dilution. The tissues were counterstained for $1 \mathrm{~h}$ with BODIPY 558/568 C12 [4, 4-difluoro-5-(2-thienyl)-4-bora-3a, 4a-diaza-s-indacene3 -dodecanoic acid, $5 \mu \mathrm{M}$; Invitrogen] to visualize adipocytes and with Hoechst 33342 ( $40 \mu \mathrm{M}$; Invitrogen) to visualize the nuclei. The tissue samples were excited using 3 laser lines (405, 488, and $568 \mathrm{~nm}$ ), and the emission was collected through appropriate narrow band-pass filters. Stacks of images (2- to $5-\mu \mathrm{m}$ thick) were collected at $0.5-\mu \mathrm{m}$ intervals along the optical axis. Each image was produced from an average of 8 frames using IpLab software version 3.6 (Scanalytics), after which the acquired images were processed using VoxBlast software (Vaytek) to produce a surface-rendered 3-dimensional model.

To identify apoptotic and necrotic cells, living tissues were incubated with YO-PRO1 (0.2 $\mu \mathrm{M}$; Invitrogen) and PI ( $0.3 \mu \mathrm{g} / \mathrm{ml}$; Invitrogen) for $20 \mathrm{~min}$, without fixation. YO-PRO1 can enter cells once their plasma membrane has reached a certain degree of permeability during apoptosis, while PI can only cross the cell membrane of necrotic cells (52). The cell membrane of viable cells is impermeable to both. YO-PRO $1^{+}$cells thus include both apoptotic and necrotic cells, while the $\mathrm{PI}^{+}$cells are necrotic. The tissue was counterstained with BODIPY and Hoechst and visualized as described above.

Analysis of hypoxia. To detect hypoxia in epididymal fat pads, immunohistochemical staining of pimonidazole adducts was performed using a hypoxyprobe-I Plus kit (Chemicon International). Mice were intraperitoneally injected with $40 \mathrm{mg} / \mathrm{kg}$ pimonidazole $1 \mathrm{~h}$ before they were 
sacrificed; hypoxic cells activate and bind the injected compounds. Adipose tissue was immediately removed and fixed, and immunohistochemical detection of pimonidazole was carried out according to the instructions provided by the manufacturer. Hypoxic cells, including adipocytes and stromal cells, were visualized, and the signal intensity of the anti-pimonidazole antibody was quantified in randomly selected fields. The tissues were also counterstained with BODIPY and Hoechst.

Isolation of the SVF and flow cytometry. To isolate the SVF, minced adipose tissue was centrifuged to remove blood cells and then incubated in a collagenase solution ( $2 \mathrm{mg} / \mathrm{ml}$, collagenase type 2; Worthington) for $30 \mathrm{~min}$. The tissue was then centrifuged, and the resultant pellet containing the SVF was filtered through $70-\mu \mathrm{m}$ mesh, incubated for $10 \mathrm{~min}$ in erythrocyte-lysing buffer, and finally resuspended in PBS. The cells were then incubated with either labeled monoclonal or isotype control antibody and analyzed by flow cytometry using a FACSCalibur flow cytometer and CellQuest Pro software (BD). The antibodies used were as follows: antiCD54 (ICAM-1, clone YN1/1.7.4; eBioscience), anti-L-selectin (CD62L, clone MEL-14; eBioscience), anti-CD11c (clone N148; eBioscience), antiPSGL1 (clone 2PH1; Research Diagnostics Inc.), anti-CD18 (clone M18/2; eBioscience), anti-E-selectin (clone 10E9.6; BD), anti-P-selectin (clone RB40.34; BD), and anti-VCAM-1 (CD106, clone 429; eBioscience).

Platelet P-selectin and lenkocyte-platelet aggregates examined by flow cytometry. A direct immunofluorescence technique was used to determine platelet activity in local resident and systemic blood using FITC-conjugated anti-CD62P (P-selectin). To obtain local blood resident in adipose tissue, fat pads were excised, minced in PBS with citrate, and centrifuged. Systemic blood was obtained by cardiac puncture. Tissue suspensions were centrifuged, and the resultant pellet was filtered through $50-\mu \mathrm{m}$ mesh. Cell suspensions were incubated with either labeled monoclonal or isotype control antibody. Leukocyte-platelet aggregates from systemic and local adipose tissue blood were analyzed. To examine the conjugates, cell suspensions were incubated with R-phycoerythrin-conjugated anti-CD41 (clone MWReg30; BD) and Alexa Fluor 488-conjugated antiF4/80 (clone BM8; Caltag).

Real-time quantitative PCR. The SVF and whole adipose tissue were homogenized in TRIzOL, and total RNA was isolated. Relative mRNA levels were calculated using the comparative Ct method; ribosomal 18S RNA served as the endogenous control. The sequences of the sense and antisense primers were

1. Hotamisligil, G.S. 2006. Inflammation and metabolic disorders. Nature. 444:860-867.

2. Suganami, T., Nishida, J., and Ogawa, Y. 2005. A paracrine loop between adipocytes and macrophages aggravates inflammatory changes: role of free fatty acids and tumor necrosis factor alpha. Arterioscler. Thromb. Vasc. Biol. 25:2062-2068.

3. Cinti, S., et al. 2005. Adipocyte death defines macrophage localization and function in adipose tissue of obese mice and humans. J. Lipid Res. 46:2347-2355.

4. Lumeng, C.N., Bodzin, J.L., and Saltiel, A.R. 2007. Obesity induces a phenotypic switch in adipose tissue macrophage polarization. J. Clin. Invest. 117:175-184.

5. Falati, S., Gross, P., Merrill-Skoloff, G., Furie, B.C., and Furie, B. 2002. Real-time in vivo imaging of platelets, tissue factor and fibrin during arterial thrombus formation in the mouse. Nat. Med. 8:1175-1181.

6. Norman, K. 2005. Techniques: Intravital microscopy - a method for investigating disseminated intravascular coagulation? Trends Pharmacol. Sci. 26:327-332.

7. Nishimura, S., et al. 2007. Adipogenesis in obesity requires close interplay between differentiating adipocytes, stromal cells, and blood vessels. Diabetes. 56:1517-1526.

as follows: P-selectin sense, 5'-TGTATCCAGCCTCTTGGGCATTCC-3'; antisense, 5'-TGGGACAGGAAGTGATGTTACACC-3'; ICAM-1 sense, 5'-ACAGACACTAGAGGAGTGAGCAGG-3'; antisense, 5'-GTGAGCGTCCATATTTAGGCATGG-3'; E-selectin sense, 5'-GGCATGTGGAATGACGAGAG-3'; antisense, 5'-TGACCACTGCAGGATG-3'; HIF-1 $\alpha$, sense, 5'-GGACAAGTCACCACAGGA-3'; antisense, 5'-GGAGAAAATCAAGTCGTG-3'.

Statistics. Results are expressed as mean \pm SEM. The statistical significance of differences between 2 groups was determined using Student's $t$ tests; differences among 3 groups were evaluated using ANOVA followed by posthoc Bonferroni tests. $P$ values less than 0.05 were considered significant.

\section{Acknowledgments}

The authors gratefully acknowledge Makoto Koyanagi for assessing the results of FACS analysis, Hiroshi Sakaue for valuable discussion on adipose tissue inflammation, and Akiko Matsuoka and Ayumi Hirano for excellent technical assistance. This study was supported by Research Fellowships from the Japan Society for the Promotion of Science for Young Scientists (S. Nishimura); by the Cell Science Research Foundation (S. Nishimura); by a Research Award on Molecular and Cellular Cardiology (S. Nishimura); by the Mochida Memorial Foundation for Medical and Pharmaceutical Research (S. Nishimura); by the Japan Diabetes Foundation (S. Nishimura); by the Vehicle Racing Commemorative Foundation (S. Sugiura); by the Nakatani Electronic Measuring Technology Association of Japan (S. Sugiura); by Grants-in-Aid for Scientific Research from the Ministry of Education, Culture, Sports, Science and Technology of Japan (I. Manabe and R. Nagai); and by a research grant from the National Institute of Biomedical Innovation (R. Nagai).

Received for publication July 19, 2007, and accepted in revised form November 28, 2007.

Address correspondence to: Satoshi Nishimura or Ichiro Manabe, Department of Cardiovascular Medicine, University of Tokyo, Hongo 7-3-1, Bunkyo-ku, Tokyo 113-0033, Japan. Phone: 81-33815-5411; Fax: 81-3-3814-0021; E-mail: snishi-tky@umin.ac.jp (S. Nishimura); manabe-tky@umin.ac.jp (I. Manabe).

8. Kumar, V., Fausto, N., and Abbas, A. 2005. Acute and chronic inflammation. In Robbins \& Cotran pathologic basis of disease. 7 th edition. V. Kumar, N. Fausto, and A. Abbas, editors. W.B. Saunders. Philadelphia, Pennsylvania, USA. 47-86.

9. Hatoum, O.A., Heidemann, J., and Binion, D.G. 2006. The intestinal microvasculature as a therapeutic target in inflammatory bowel disease. Ann. N. Y. Acad. Sci. 1072:78-97.

10. Blankenberg, S., Barbaux, S., and Tiret, L. 2003. Adhesion molecules and atherosclerosis. Atherosclerosis. 170:191-203.

11. Miller, M.A., and Cappuccio, F.P. 2006. Cellular adhesion molecules and their relationship with measures of obesity and metabolic syndrome in a multiethnic population. Int. J. Obes. (Lond.) 30:1176-1182

12. Basili, S., et al. 2006. Insulin resistance as a determinant of platelet activation in obese women. J. Am. Coll. Cardiol. 48:2531-2538.

13. Kampf, C., Bodin, B., Kallskog, O., Carlsson, C., and Jansson, L. 2005. Marked increase in white adipose tissue blood perfusion in the type 2 diabetic GK rat. Diabetes. 54:2620-2627.

14. Simonsen, L., Enevoldsen, L.H., and Bulow, J. 2003. Determination of adipose tissue blood flow with local 133Xe clearance. Evaluation of a new labelling technique. Clin. Physiol. Funct. Imaging. 23:320-323.
15. Downey, G.P., et al. 1990. Retention of leukocytes in capillaries: role of cell size and deformability. J. Appl. Physiol. 69:1767-1778.

16. Eppihimer, M.J., and Lipowsky, H.H. 1994. Leukocyte sequestration in the microvasculature in normal and low flow states. Am. J. Physiol. 267:H1122-H1134.

17. Ley, K. 2003. The role of selectins in inflammation and disease. Trends Mol. Med. 9:263-268.

18. Robker, R.L., Collins, R.G., Beaudet, A.L., Mersmann, H.J., and Smith, C.W. 2004. Leukocyte migration in adipose tissue of mice null for ICAM-1 and Mac-1 adhesion receptors. Obes. Res. 12:936-940.

19. Zarbock, A., Polanowska-Grabowska, R.K., and Ley, K. 2007. Platelet-neutrophil-interactions: Linking hemostasis and inflammation. Blood. Rev. 21:99-111.

20. Michelson, A.D., Barnard, M.R., Krueger, L.A., Valeri, C.R., and Furman, M.I. 2001. Circulating monocyte-platelet aggregates are a more sensitive marker of in vivo platelet activation than platelet surface P-selectin: studies in baboons, human coronary intervention, and human acute myocardial infarction. Circulation. 104:1533-1537.

21. Huo, Y., et al. 2003. Circulating activated platelets exacerbate atherosclerosis in mice deficient in apolipoprotein E. Nat. Med. 9:61-67. 
22. Dubois, C., Panicot-Dubois, L., Gainor, J.F., Furie, B.C., and Furie, B. 2007. Thrombin-initiated platelet activation in vivo is $\mathrm{VWF}$ independent during thrombus formation in a laser injury model. J. Clin. Invest. 117:953-960.

23. Wiernsperger, N., Nivoit, P., De Aguiar, L.G., and Bouskela, E. 2007. Microcirculation and the metabolic syndrome. Microcirculation. 14:403-438.

24. Yuan, S.Y., et al. 2007. Microvascular permeability in diabetes and insulin resistance. Microcirculation. 14:363-373.

25. Weisberg, S.P., et al. 2003. Obesity is associated with macrophage accumulation in adipose tissue. J. Clin. Invest. 112:1796-1808.

26. Xu, H., et al. 2003. Chronic inflammation in fat plays a crucial role in the development of obesity-related insulin resistance. J. Clin. Invest. 112:1821-1830.

27. Fan, J., and Watanabe, T. 2003. Inflammatory reactions in the pathogenesis of atherosclerosis. J. Atheroscler. Thromb. 10:63-71.

28. Moulton, K.S., et al. 2003. Inhibition of plaque neovascularization reduces macrophage accumulation and progression of advanced atherosclerosis. Proc. Natl. Acad. Sci. U. S. A. 100:4736-4741.

29. Ross, R. 1999. Atherosclerosis - an inflammatory disease. N. Engl. J. Med. 340:115-126.

30. Majno, G. 1998. Chronic inflammation: links with angiogenesis and wound healing. Am. J. Pathol. 153:1035-1039.

31. Giannelli, G., Quaranta, V., and Antonaci, S. 2003. Tissue remodelling in liver diseases. Histol. Histopathol. 18:1267-1274.

32. Chavey, C., et al. 2003. Matrix metalloproteinases are differentially expressed in adipose tissue during obesity and modulate adipocyte differentiation. J. Biol. Chem. 278:11888-11896.

33. Maquoi, E., Munaut, C., Colige, A., Collen, D., and Lijnen, H.R. 2002. Modulation of adipose tissue expression of murine matrix metalloproteinases and their tissue inhibitors with obesity. Diabetes. 51:1093-1101.

34. Demeulemeester, D., Collen, D., and Lijnen, H.R. 2005. Effect of matrix metalloproteinase inhibition on adipose tissue development. Biochem. Biophys. Res. Commun. 329:105-110.

35. Lijnen, H.R., et al. 2002. Matrix metalloproteinase inhibition impairs adipose tissue development in mice. Arterioscler. Thromb. Vasc. Biol. 22:374-379.

36. Pierleoni, C., Verdenelli, F., Castellucci, M., and Cinti, S. 1998. Fibronectins and basal lamina molecules expression in human subcutaneous white adipose tissue. Eur. J. Histochem. 42:183-188.

37. Cancello, R., and Clement, K. 2006. Is obesity an inflammatory illness? Role of low-grade inflammation and macrophage infiltration in human white adipose tissue. BJOG. 113:1141-1147.

38. Sell, H., Dietze-Schroeder, D., and Eckel, J. 2006. The adipocyte-myocyte axis in insulin resistance. Trends Endocrinol. Metab. 17:416-422.

39. Fox, C.S., et al. 2007. Abdominal visceral and subcutaneous adipose tissue compartments: association with metabolic risk factors in the Framingham Heart Study. Circulation. 116:39-48.

40. Savill, J., and Fadok, V. 2000. Corpse clearance defines the meaning of cell death. Nature. 407:784-788.

41. Ravichandran, K.S. 2003. "Recruitment signals" from apoptotic cells: invitation to a quiet meal. Cell. 113:817-820.

42. Summers, L.K., Samra, J.S., and Frayn, K.N. 1999. Impaired postprandial tissue regulation of blood flow in insulin resistance: a determinant of cardiovascular risk? Atherosclerosis. 147:11-15.

43. Russell, J., Cooper, D., Tailor, A., Stokes, K.Y., and Granger, D.N. 2003. Low venular shear rates promote leukocyte-dependent recruitment of adher- ent platelets. Am. J. Physiol. Gastrointest. Liver Physiol. 284:G123-G129.

44. Harris, A.G., Skalak, T.C., and Hatchell, D.L. 1994. Leukocyte-capillary plugging and network resistance are increased in skeletal muscle of rats with streptozotocin-induced hyperglycemia. Int. J. Microcirc. Clin. Exp. 14:159-166.

45. Bolton, S.J., Anthony, D.C., and Perry, V.H. 1998. Loss of the tight junction proteins occludin and zonula occludens- 1 from cerebral vascular endothelium during neutrophil-induced blood-brain barrier breakdown in vivo. Neuroscience. 86:1245-1257.

46. Kurose, I., et al. 1994. Molecular determinants of reperfusion-induced leukocyte adhesion and vascular protein leakage. Circ. Res. 74:336-343.

47. Williams, K.J., and Tabas, I. 2005. Lipoprotein retention - and clues for atheroma regression. Arterioscler. Thromb. Vasc. Biol. 25:1536-1540.

48. Kumasaka, T., et al. 1996. Role of the intercellular adhesion molecule-1(ICAM-1) in endotoxininduced pneumonia evaluated using ICAM- 1 antisense oligonucleotides, anti-ICAM-1 monoclonal antibodies, and ICAM-1 mutant mice. J. Clin. Invest. 97:2362-2369.

49. Jones, S.P., Girod, W.G., Granger, D.N., Palazzo, A.J., and Lefer, D.J. 1999. Reperfusion injury is not affected by blockade of P-selectin in the diabetic mouse heart. Am. J. Physiol. 277:H763-H769.

50. Ramos, C.L., et al. 1997. Differential effect of E-selectin antibodies on neutrophil rolling and recruitment to inflammatory sites. Blood. 89:3009-3018.

51. Harris, A.G., Sinitsina, I., and Messmer, K. 2002. Intravital fluorescence microscopy and phototocicity: effects on leukocytes. Eur. J. Med. Res. 7:117-124.

52. Mammone, T., et al. 2000. Successful separation of apoptosis and necrosis pathways in $\mathrm{HaCaT}$ keratinocyte cells induced by UVB irradiation. Cell Biol. Toxicol. 16:293-302. 